\title{
La mobilité des personnes âgées dans le « quartier durable » de Rieselfeld à Fribourg-en-Brisgau
}

Mobility of elderly people in the "sustainable neighborhood" of Freiburg-

Rieselfeld

Mobilität älterer Menschen im "nachhaltigen Quartier" Rieselfeld in Freiburg im Breisgau

Hassina Imerzoukene Driad, Philippe Hamman and Tim Freytag

\section{(2) OpenEdition \\ Journals}

Electronic version

URL: http://journals.openedition.org/rge/5221

DOI: $10.4000 /$ rge.5221

ISSN: 2108-6478

Publisher

Association des géographes de l'Est

Printed version

Date of publication: 30 December 2014

ISSN: 0035-3213

\section{Electronic reference}

Hassina Imerzoukene Driad, Philippe Hamman and Tim Freytag, «La mobilité des personnes âgées dans le « quartier durable » de Rieselfeld à Fribourg-en-Brisgau », Revue Géographique de l'Est [Online] vol. 54 / n`3-4 | 2014, Online since 04 February 2015, connection on 08 September 2020. URL : http:// journals.openedition.org/rge/5221 ; DOI : https://doi.org/10.4000/rge.5221

This text was automatically generated on 8 September 2020 .

Tous droits réservés 


\section{La mobilité des personnes âgées dans le « quartier durable » de Rieselfeld à Fribourg-en-Brisgau}

Mobility of elderly people in the "sustainable neighborhood" of Freiburg-

\section{Rieselfeld}

Mobilität älterer Menschen im "nachhaltigen Quartier" Rieselfeld in Freiburg im Breisgau

Hassina Imerzoukene Driad, Philippe Hamman and Tim Freytag

\section{Introduction}

1 Le concept de « quartier durable » revient aujourd'hui de façon récurrente et s'inscrit dans une nouvelle approche urbanistique pour la planification et l'étude de la ville durable. Il favorise l'expérimentation de différents dispositifs d'aménagement et peut aussi permettre d'accroître la culture participative au sein du quartier. En outre, il représente une échelle spécifique d'un projet urbain qui s'avère complémentaire à la réflexion sur le développement durable au niveau de l'agglomération (Charlot-Valdieu, 2000).

2 Par ailleurs, la mobilité constitue une valeur centrale de la modernité et une préoccupation majeure de la ville actuelle ${ }^{1}$. Comme le rappelle J.-P. Orfeuil (2004), la mobilité est considérée comme une nouvelle norme sociale, où «l'aptitude à la mobilité, la capacité de se rendre dans divers types d'espaces, parfois éloignés de son domicile ou de sa commune, sont, aujourd'hui plus qu'hier, des conditions indispensables pour la construction de trajectoires de vie satisfaisantes et de liens sociaux diversifiés ». La mobilité s'observe aussi par des pratiques de déplacement dans l'espace et le temps et renvoie à une multitude de motifs et de moyens de déplacement et surtout à l'usager, dont la mobilité est conçue à la fois comme une liberté et une obligation (Le Breton, 2005). 
3 Dans un contexte de développement durable, l'objectif est d'arriver à une «mobilité partagée » où les modes collectifs de déplacements urbains sont accessibles à tous, $\mathrm{y}$ compris aux personnes fragiles ou à mobilité réduite, telles que les personnes âgées (Hamman, 2011). Pour ces dernières, le niveau de mobilité détermine en grande partie leur accessibilité aux services et aux transports, ainsi que leur sentiment de liberté et de bien-être (Sterns, 2003). A contrario, le manque d'accès aux transports et aux services publics et les problèmes financiers créent des barrières pour ces personnes à mobilité réduite. De ce fait, la mobilité s'impose parmi les enjeux majeurs au cœur de la réflexion sur la problématique du vieillissement. L'intérêt croissant que l'on porte au comportement de mobilité des personnes âgées est lié en grande partie à la croissance continue de cette population, et qui est appelée à se poursuivre dans les prochaines décennies. Il est donc important d'identifier et d'anticiper leurs comportements et besoins en matière de transport et d'explorer l'influence du milieu de vie sur leur bienêtre (physique et psychologique).

4 Dans cette étude, partant d'un regard analytique sur la ville de Fribourg, qui est souvent tenue pour exemplaire en matière de développement durable (Beim, 2010; Buehler, 2011 ; Medearis, 2012), nous proposons, dans un premier temps, de porter une attention particulière à sa politique d'aménagement urbain. À l'image de la ville de Fribourg-en- Brisgau, le quartier de Rieselfeld est en train de se forger une certaine réputation en matière d'urbanisme durable (Humpert, 1997), en tant que système relationnel, champ d'actions transversales et terrain d'expérimentation pionnier du développement durable. Dans une seconde partie, nous examinerons plus en détail la mobilité des personnes âgées au sein de ce quartier. Cette réflexion a été notamment motivée par l'augmentation du nombre de seniors choisissant de s'installer dans le quartier.

5 Nous nous fondons empiriquement sur une enquête quantitative et qualitative (Imerzoukene, 2012) que nous avons effectuée en 2012 sur les comportements de mobilité, en interrogeant la capacité d'adaptation des personnes âgées dans le quartier de Rieselfeld à Fribourg. Dans cette optique, nous nous proposons de décliner cette problématique autour des deux questions suivantes:

-Comment l'aménagement d'un écoquartier favorable à la mobilité durable peut-il contribuer aux changements des pratiques de mobilité des personnes âgées?

- Y a-t-il eu des changements réels dans les habitudes de comportements de mobilité des personnes âgées suite à la mise en œuvre d'une politique de mobilité durable à Rieselfeld ?

Méthodologiquement, l'enquête est basée sur une vingtaine d'interviews et un questionnaire standardisé distribué aléatoirement auprès d'un échantillon de 350 personnes âgés. Pour désigner la population étudiée, plusieurs expressions courantes sont utilisées : personnes âgées, aînés, troisième âge, seniors, etc. Bien que la définition d'une personne âgée dépende du contexte, toutes ces appellations convergeant vers la même notion: la vieillesse. Cette dernière est un état résultant de l'intrication des dimensions biologiques, psychologiques et sociales d'un processus de vieillissement, qui s'est déroulé tout au long d'une vie (Coudin, 2005). En suivant la définition de l'Organisation mondiale de la santé (OMS), nous associons les personnes âgées aux plus de 60 ans. Par ailleurs, c'est aussi ce « passage » des 60 ans qui est souvent retenu pour certaines prestations ou dispositions dans les réglementations françaises et allemandes.

Dans cette enquête, nous avons mis l'accent sur les pratiques de mobilité, les motifs de déplacement et les choix respectifs de transports associés aux seniors. Des questions 
relatives aux changements dans les schémas de mobilité de ces personnes depuis leur emménagement dans le quartier, et le degré de satisfaction vis-à-vis de ce dernier et de son accessibilité ont été également considérées. Pour les interrogés, ce fut aussi l'occasion de signaler des problèmes et d'exprimer des suggestions d'amélioration, éléments significatifs pour notre analyse.

$8 \mathrm{Au}$ début de l'enquête, selon les statistiques de la ville de Fribourg (FRITZ) ${ }^{2}$, la population totale de Rieselfeld s'élevait à 9500 individus. La population âgée de 60 ans et plus représentait 860 personnes, soit $9 \%$ de la population totale du quartier. Sur les 350 questionnaires distribués aux seniors sur place, 105 réponses exploitables ont été transmises à l'association de quartier $\mathrm{KIOSK}^{3}$, correspondant à un taux de retour de $30 \%$.

\section{L'option du développement durable : l'urbanisme contre l'automobile?}

\section{A. Cadre de la politique de planification des transports dans l'optique d'une mobilité durable}

9 L'approche globale de la planification des transports dans la ville de Fribourg est guidée par des principes écologiques visant à améliorer l'environnement en réduisant essentiellement le trafic des voitures (Hecker, 2007). Déjà, après la Deuxième Guerre mondiale, plutôt que de suivre la tendance des modèles de l'époque orientés vers la voiture et basés sur des faibles densités de développement et sur l'étalement urbain, la planification de la ville a suivi un modèle traditionnel plutôt compact, où il ne s'agissait pas seulement d'offrir un cadre de vie riche et pluriel, mais aussi de lutter contre les déplacements motorisés ${ }^{4}$.

10 La politique de développement urbain général et la politique des transports ont été intimement imbriquées et considérées comme une unité. Des résultats marquants dans les « déviations » à l'intérieur de la répartition modale ne peuvent, toutefois, émerger qu'après une certaine période de temps. Bien que cela ait demandé deux décennies pour que la dynamique " prenne " véritablement, le recul de l'utilisation automobile en faveur des déplacements doux est aujourd'hui net à Fribourg 5 .

11 Le premier plan intégré de la circulation (1969) a donné une égale priorité aux différents modes de transport (piétons, vélos, transports collectifs et circulation des véhicules). Il a incité à l'utilisation croissante du vélo, qui a pris une place importante dans la politique locale des transports. Entre 1982 et 1999, la contribution du vélo au trafic de la ville est passée de 15 à $27 \%$. Dans le même temps, la part des transports en commun a progressé de 11 à $18 \%$, tandis que la part des individus conducteurs et passagers, utilisant des véhicules à moteur a diminué de 38 à $30 \%{ }^{6}$.

Depuis 1990, dans le but de protéger les piétons et les cyclistes, $90 \%$ des zones résidentielles ont été converties en zones à $30 \mathrm{~km} / \mathrm{h}$. Un concept de gestion continue de l'espace de stationnement a été également mis en place dans de nombreuses parties de la ville (au centre-ville, toutes les places de parking sont payantes).

13 La réduction sensible de l'utilisation des voitures a été aussi rendue possible grâce à différentes mesures. Parmi celles-ci, l'introduction en 1984 d'un ticket régional mensuel appelé «Umweltkarte» (carte environnementale) permettant de circuler de 
manière illimitée sur tout le réseau de transport collectif de la ville (FitzRoy, 1998). Fribourg a d'ailleurs été l'une des premières villes en Allemagne à introduire un ticket de transport transférable à d'autres utilisateurs. Ce coupon a été remplacé en 1991 par une carte régionale de transport : (Regio-Karte), permettant d'accéder sans limites à tous les transports collectifs (trains, bus, tramways, etc.) régionaux et urbains (dans un rayon de $60 \mathrm{~km}$ autour de la ville). Les avantages de cette carte ont déclenché une augmentation très nette du nombre de passagers dans les transports en commun.

Concernant la répartition modale des personnes âgées à l'échelle de la ville, et selon un rapport d'analyse de la ville de Fribourg ${ }^{7}$, il apparait que l'utilisation des transports en commun augmente sensiblement après 59 ans. Cependant, le recours à la voiture personnelle par les seniors reste relativement important jusqu'à 70 ans, même s'il montre un certain recul par rapport aux populations plus jeunes. Par contre, à partir de 70 ans, l'usage des transports en commun augmente et l'utilisation de la voiture et du vélo baissent considérablement.

À travers l'exemple de Fribourg, il apparaît que les caractéristiques de la mobilité, qui est de nos jours largement dominée par l'automobile, peuvent être au moins en partie orientées en faveur d'une mobilité durable grâce à des outils d'aménagement du territoire et de transports.

\section{B. Le quartier durable : facette de l'urbanisme durable}

16 À la fin des années 1980, Fribourg a fait face à une forte pénurie de logements, ce qui a motivé la construction de nouveaux quartiers. Les nouveaux quartiers durables de Rieselfeld et Vauban sont souvent cités comme exemples de réussite de la politique de développement urbain de Fribourg. En particulier, le quartier durable de Rieselfeld, distant d'environ $5 \mathrm{~km}$ du centre-ville (cf. figure 1a), a constitué l'un des plus grands projets de constructions nouvelles du Land de Bade-Wurtemberg.

17 Avant sa construction, Rieselfeld était un terrain isolé, d'une superficie de 78 ha: il tient son nom d'anciens terrains d'épuration et de décantation des eaux usées de la partie sud-ouest de Fribourg. Après un certain nombre d'études approfondies du sol et différentes mesures d'assainissement, le quartier a été déclaré urbanisable à partir de 1985, notamment en zone résidentielle. L'ensemble du quartier est bordé par une réserve naturelle protégée (plus de 200 hectares) et se situe à proximité d'un parc animalier (Mundenhof), accessible à partir de Rieselfeld uniquement à pied ou à vélo. Cette préservation environnementale correspond à un engagement de la municipalité pour que les constructions ne se développent pas trop aux dépens du biotope reconnu de ce site ${ }^{8}$. 
Figure 1 : Situation géographique du quartier Rieselfeld (a) et le plan correspondant des équipements (b)
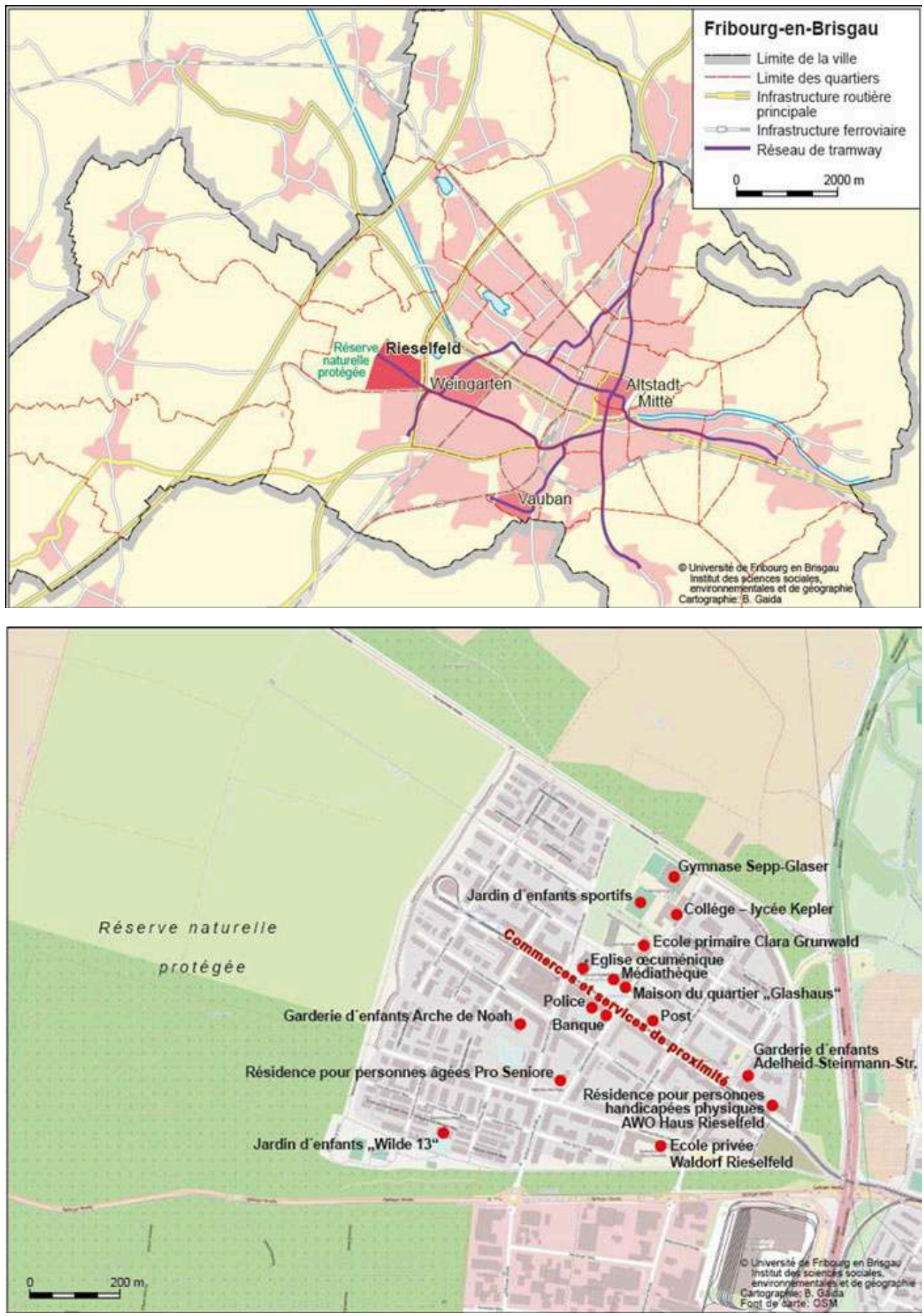

\section{Un projet d'urbanisme durable évolutif}

Le projet a été élaboré de manière à éviter les erreurs des grands ensembles des années 1950-1970. Raison pour laquelle une concertation étroite entre la ville, les futurs habitants et les bureaux d'étude a été entreprise en amont du projet. Un cahier des charges avait défini des exigences strictes en termes de performance énergétique, de modularité de l'habitat, d'insonorisation, d'aménagements, etc. 
19 Les premiers habitants de Rieselfeld sont arrivés en 1996, et la mise en service du tramway est effectuée juste une année après. L'organisation du quartier (cf. figure 1b) devait s'articuler autour d'une ligne de tramway qui assure la liaison avec le centreville de Fribourg (cf. figures 1a,b). Cet axe forme une avenue structurante pour l'ensemble des activités économiques. En outre, le projet permet de dégager de multiples espaces verts et une organisation des déplacements, qui accorde la priorité aux modes doux et aux transports publics: vitesse limitée à $30 \mathrm{~km} / \mathrm{h}$, zones sans stationnement, transports en commun en site propre, pistes cyclables et arrêts de tramway avec parkings pour vélos.

La vision prospective des concepteurs a permis de construire des équipements publics durables parce que modulaires et modulables dans le temps. Son plan présentait une souplesse dans la programmation, qui permettait d'introduire des modifications selon les développements futurs, prenant en compte les besoins des familles ainsi que des personnes à mobilité réduite. En outre, des cours urbaines au cœur d'îlots ouverts forment des espaces semi-privés, qui permettent la transition entre l'espace public et l'espace privé au sein du quartier.

21 Le quartier de Rieselfeld a aussi été conçu avec l'objectif de rapprocher les habitants des lieux de travail et des commerces (mixité des fonctions urbaines, quartier à courtes distances). Le but étant de réduire le nombre de déplacements pendulaires, la priorité est donnée dans tous les cas aux piétons et aux vélos. Comme la majorité des rues sont résidentielles, la vitesse est limitée dans tout le quartier à $30 \mathrm{~km} / \mathrm{h}$.

La rue principale (Rieselfeldallee) comporte deux voies de circulation parallèles et à sens unique, séparées par la ligne de tramway. Des immeubles d'habitation et des équipements commerciaux (magasins, boulangerie, cafés et restaurants, studio photo, salon de coiffure), ainsi qu'une grande variété de services (banque, poste, police, médecins, dentistes, pharmacie, centre de rééducation, salles de gym, espace culturel, agences de voyages, etc.) ont progressivement vu le jour. En outre, du fait de la dépendance directe de ces implantations de la demande et de l'évolution démographique du quartier, les derniers terrains libres de la Rieselfeldallee n'ont été bâtis qu'à la fin du projet (discount Lidl, centre commercial, habitations, bureaux, etc.). Le quartier dispose également d'un ensemble d'infrastructures sociales (écoles, garderies, maison de quartier, gymnase, église, maison de retraite, etc.) nécessaires pour répondre aux besoins de tous ses habitants. La réserve naturelle, à vocation d'espace de détente, jouxte directement le quartier Rieselfeld et demeure protégée.

24 Une enquête, mandatée par le groupe de projet Rieselfeld en 2010, a été réalisée par l'Institut des sciences sociales appliquées de Fribourg (FIFAS ${ }^{9}$ ) afin de dresser un bilan provisoire de l'avancement du projet. Les résultats obtenus confirment la satisfaction de l'ensemble des habitants interrogés à l'égard du développement de leur quartier (Schings, 2011).

Le travail qui a été mené avec la même attention dans les domaines social, culturel (association K.I.O.S.K), écologique et architectural a largement contribué à ce résultat. Une image positive, des infrastructures complètes adaptées aux besoins des habitants et une vie sociale active sont autant d'éléments qui motivent aussi bien les jeunes familles que les personnes âgées à rester ou à venir s'installer à Rieselfeld. L'attrait et la popularité du quartier sont statistiquement confirmés par l'augmentation continue du 
nombre total d'habitants. Au début de 1996, alors que seuls 62 individus vivaient à Rieselfeld, ce nombre est passé à 6810 à la fin 2004, et 9500 en janvier $2012^{10}$.

Comme pour la plupart des localités en Allemagne, le quartier de Rieselfeld est confronté au défi de l'intégration sociale d'effectifs sans cesse croissants de personnes âgées. $\mathrm{Si}$, à l'heure actuelle, Rieselfeld représente l'un des quartiers où résident le moins de seniors à l'échelle de la ville de Fribourg (860 personnes de 60 ans ou plus à la fin 2011, soit 9\% de la population totale du quartier Rieselfeld), il lui faudra gérer le vieillissement de cette tranche de population, qui sera rattrapée par les générations des plus jeunes (la population des plus de 40/50 ans correspondant actuellement aux cohortes majoritaires du quartier s'élevait à près de 2000 personnes au 1er janvier 2012). Selon le service des statistiques de la ville de Fribourg, l'allongement de la durée de vie va alourdir le poids démographique des plus de 60 ans dans la structure sociale du quartier. À l'horizon 2025, leur nombre devrait même plus que tripler, pour passer de 860 à plus de 2800 personnes $^{11}$.

\section{Quels sont les comportements de mobilité des personnes âgées à Rieselfeld ?}

\section{A. Profil des personnes âgées interrogées}

La structure de la population des personnes âgées interrogées par questionnaire est caractérisée par une part importante des classes de populations âgées de 65 à 75 ans (cf. figure 2). La moyenne d'âge pour les hommes est de 71 ans et celle des femmes de 72 ans. Globalement, les femmes représentent $60 \%$ de notre échantillon de seniors. Sans surprise, on remarque également sur la figure 2 que le pourcentage des octogénaires et nonagénaires est constitué en majorité par des femmes.

Figure 2 : Pyramide des âges des personnes interrogées selon l'âge et le sexe

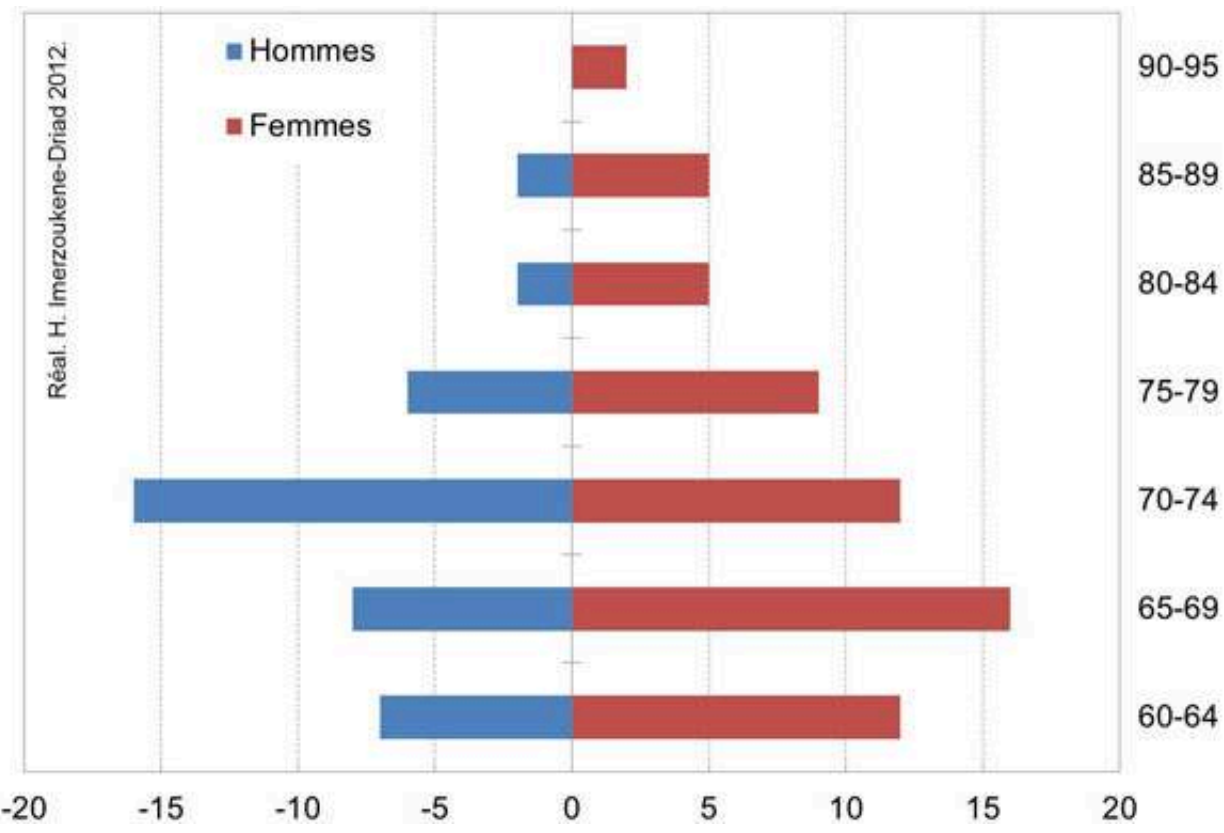


28 Le dépouillement des réponses montre également que plus de la moitié des personnes entre 60 et 80 ans vivent en couple. Plus d'un tiers de l'échantillon interrogé vit seul (célibataire ou veuf). Les femmes veuves représentent plus de la moitié de ces personnes, du fait que le veuvage touche généralement plus les femmes que les hommes.

29 Au regard de l'enquête menée, il ressort que le problème qui inquiète et qui génère le plus de craintes pour les personnes âgées ne semble pas être celui de la mobilité en priorité, mais la santé. Comme l'ont noté Alsnih et Hensher (2003), les capacités fonctionnelles des personnes diminuent avec l'âge et les gênes ressenties lors des déplacements peuvent conditionner et affecter la mobilité. Toutefois, lors de nos entretiens, notamment à la résidence "Pro Seniore", les personnes ne pouvant se déplacer seules ont signalé qu'elles essaient quand même de sortir avec le soutien de leur famille, même si ce n'est que pour parcourir des distances relativement courtes.

30 Confirmant la littérature sur le sujet (Caradec, 2007 ; Negron-Poblete, 2012), on observe des stratégies de déplacement pour atténuer le déclin des compétences individuelles et une adaptation des habitudes, afin de surmonter tant bien que mal les difficultés à se déplacer. En effet, la majorité des seniors essayent de s'adapter malgré tout à leur situation et de « rebondir » face aux vicissitudes de la vie, plutôt que de s'abandonner à une vieillesse domestique prématurée.

\section{B. Rieselfeld est-il attractif pour les personnes âgées?}

On l'a vu, le quartier de Rieselfeld est un quartier relativement jeune (l'installation des premiers habitants remonte à 1996). Il n'était constitué à ses débuts que de jeunes familles, avec une quasi-absence de personnes âgées. Mais, avec le temps, les données ont sensiblement changé. D'après les résultats de notre enquête et en correspondance avec les statistiques démographiques de la ville ${ }^{12}$, il apparaît que le nombre de seniors vivant dans le quartier ne cesse d'augmenter au fil du temps. En effet, alors que le quartier ne comptait aucun senior en 1995, il accueille près de 200 personnes âgées (>59 ans) en 2000, pour atteindre plus de 860 seniors au 1er janvier 2012. Ceci peut être clairement observé sur la figure 3, qui reflète l'augmentation rapide de la population âgée dans le quartier. 
Figure 3 : Évolution démographique de la population de personnes âgées à Rieselfeld

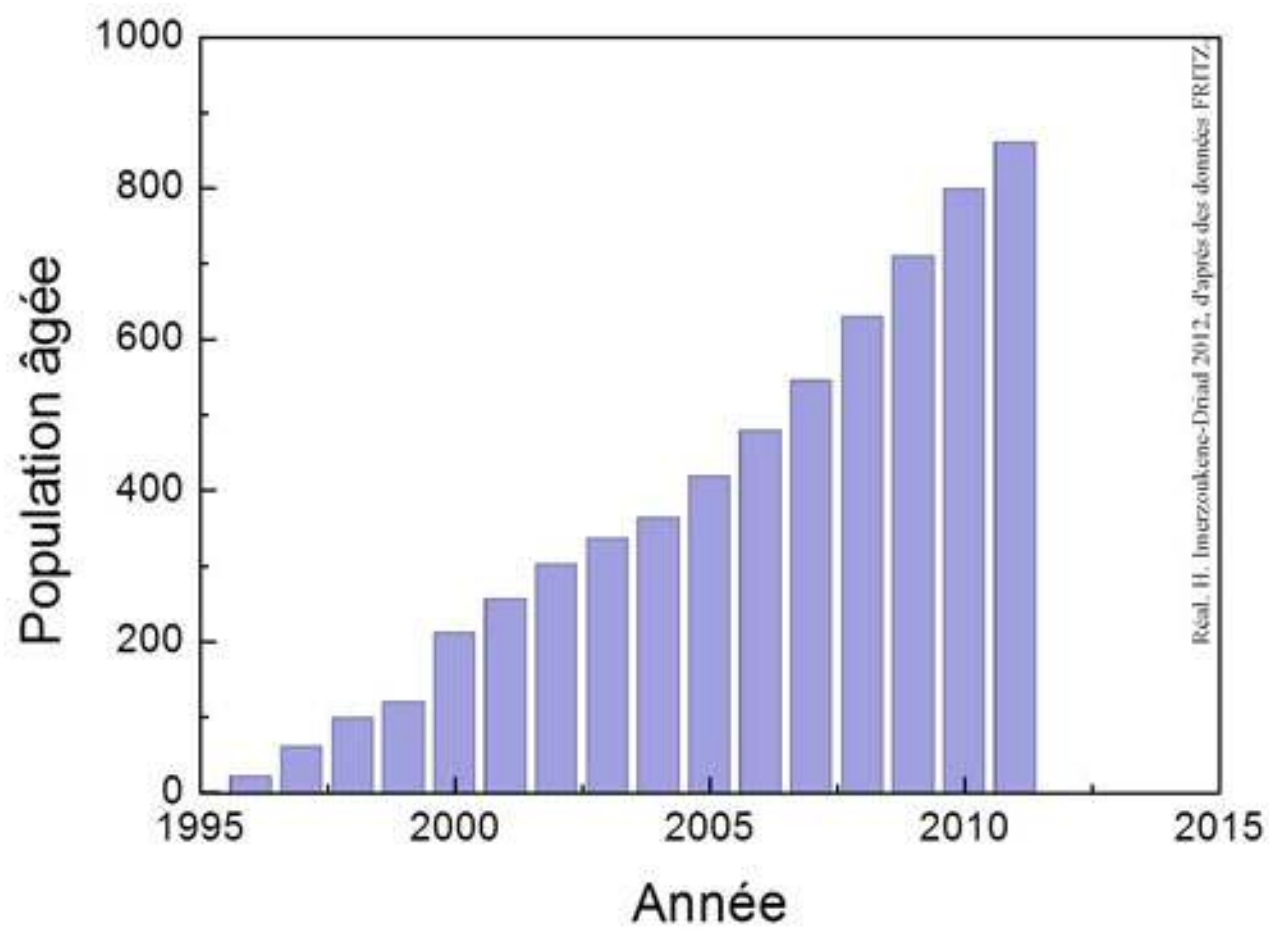

source : Statistiques « en ligne » de la ville de Fribourg

En premier lieu, on peut estimer que l'effectif des seniors dans le quartier peut résulter du vieillissement de la population locale. À partir des réponses aux questionnaires, nous avons d'abord collecté l'âge actuel des seniors (période de l'enquête) et leur date d'installation dans le quartier. Ensuite, nous avons déduit l'âge de ces personnes à l'arrivée dans le quartier (Âge à l'arrivée = Âge actuel - [2012 - Année d'arrivée]). À titre d'exemple, la personne la plus âgée du quartier, qui avait 94 ans en 2012, est arrivée en 2002, à l'âge de 84 ans. Il est à noter que l'âge à l'arrivée dans le quartier peut influer sur le mode de socialisation ou le choix des modes de déplacements. Après analyse des résultats (cf. figure 4), il nous est apparu que la majorité des personnes interrogées est arrivée soit juste avant, soit juste après l'âge de la retraite (60-65 ans). Par ailleurs, il est intéressant de noter que certains seniors ont décidé de s'installer dans le quartier même à un âge relativement avancé. 
Figure 4 : Graphique de la répartition des seniors selon leur âge à l'arrivée dans le quartier de Rieselfeld

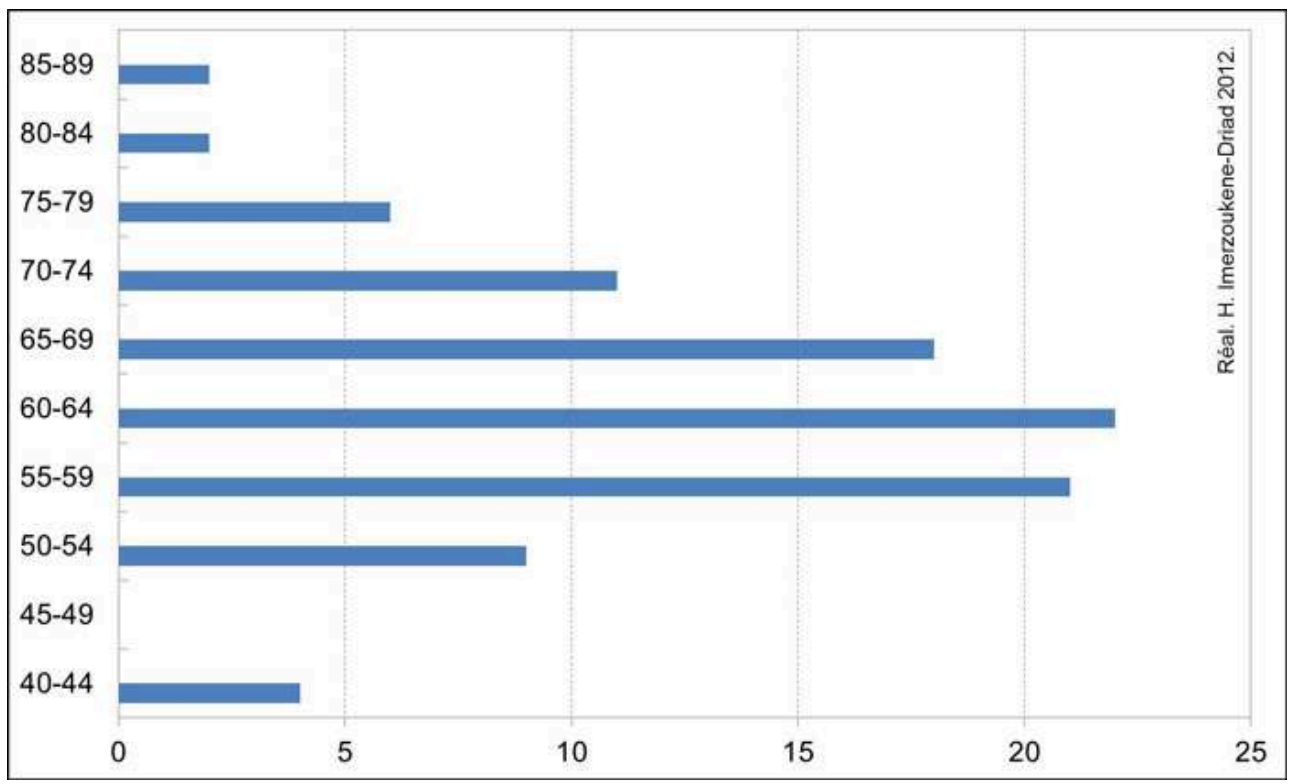

Une explication à cette tendance ressort des propos recueillis lors d'un entretien avec Monsieur K. Siegl (chef du projet Rieselfeld, aujourd'hui à la retraite) :

"Le quartier était conçu dès le départ pour convenir aussi bien aux personnes âgées qu'aux familles avec enfants. Il se voulait comme quartier intergénérationnel, en favorisant la création de liens sociaux entre les générations à l'aide d'espaces communs et des services qui répondent aux besoins de tout le monde. L'urbanisation du quartier s'est faite en même temps que l'arrivée des premiers habitants (construction des logements, réalisation du réseau viaire, etc.). C'était un quartier en chantier avec tous les inconvénients que cela comporte (les grandes grues, bruit, poussière, livraison des matériaux, etc.). Au début, les habitants étaient donc composés dans leur totalité de jeunes familles, qui ont déménagé à Rieselfeld et ont assisté à sa construction ${ }^{\circ}$.

Les personnes âgées qui disposaient de logements ailleurs, et a priori pour des raisons de commodité, avaient moins envie de déménager dans un quartier en construction (travaux, bruit, etc.). De plus, la crainte qu'avaient la plupart d'entre elles d'habiter à proximité d'un ancien quartier ayant connu des problèmes sociaux (FribourgWeingarten) les avait découragées au départ de s'installer dans le quartier. En outre, cette population pouvait avoir des appréhensions par rapport au site de Rieselfeld, utilisé par le passé comme décharge. Avec le temps, et à travers la réputation de durabilité que s'est progressivement forgée le quartier, un nombre de plus en plus important de personnes âgées s'est décidé à s'y installer.

Ce constat est également confirmé par les observations et commentaires rapportés dans nos questionnaires ou lors de nos entretiens effectués avec certains résidents : «Le quartier est agréable à vivre ». "Tout est bien conçu et organisé. Rieselfeld est bien adapté pour tous les comportements de mobilité ». "L'offre en magasins et autres services est bonne, il $y$ a tout à Rieselfeld ». «Le quartier est bien connecté au reste de la ville, c'est très pratique ». " Le quartier dispose de grands espaces ouverts, des rues larges,...».

38 Par ailleurs, les entretiens menés avec des personnes âgées montrent que celles-ci intègrent une représentation du quartier, dans leurs modes de vie et logiques de déplacement, qui leur est propre. Elles élaborent des stratégies de réaménagement 
d'existence - dont les fondements sont les habitudes quotidiennes et certaines formes de ritualisation dans leurs pratiques de mobilité - qui les rassurent et qui les maintiennent mobiles (par exemple, pour l'approvisionnement et les loisirs), comme le traduit le témoignage de Madame N. S (87 ans) :

"J'essaye de marcher tous les jours. C'est très important pour la santé... Je fais mes courses dans le quartier, des fois, je vais m'asseoir dans le parc où il y a les rosiers. Je rencontre quelques vieilles dames comme moi et on discute.... Parfois, ça m'arrive aussi de me déplacer jusqu'à la maison du quartier (Glashaus) pour assister aux programmes pour seniors (Seniore-café, cinéma, rencontres)...».

En outre, l'association K.I.O.S.K entreprend aussi différentes initiatives, dans le cadre des projets pour les personnes âgées, qui consistent à monter des activités et à organiser des marches et des rencontres entre les seniors ${ }^{13}$. Pour nombre d'entre eux, ces occasions représentent déjà des motifs pour sortir de leur domicile, marcher et participer à la vie sociale du quartier.

41 À la question de savoir si les seniors «avaient déjà pensé quitter le quartier à cause de leur situation actuelle », $90 \%$ des interrogés ont répondu par la négative. La grande majorité souhaite rester dans le quartier et conserver les relations sociales nouées autour de leur lieu de vie. Pour confirmer leur satisfaction, certains font même remarquer qu'ils ont déménagé à Rieselfeld à cause de l'âge et que le quartier dispose des structures adaptées à leur situation (résidence pour seniors, commerces de proximité et offre suffisante de transports). Pour les $10 \%$ de seniors ayant émis des réserves, les raisons avancées sont d'ordre familial ou économique (cherté de la vie à Rieselfeld). En effet, lors de notre enquête, l'augmentation du loyer a été souvent citée parmi les raisons qui pourraient pousser certains seniors à quitter Rieselfeld. Ces éléments incitent à se poser des questions sur le devenir de la mixité sociale au sein du quartier et risqueraient même de remettre en cause les principes et les efforts consentis pour instaurer un quartier durable.

\section{Quels sont les moyens, les motifs et les lieux de déplacement des personnes âgées?}

L'augmentation de la motorisation chez les personnes âgées a été relevée dans de nombreuses études (Pochet, 1996) ${ }^{14}$. La tendance des seniors à garder leurs voitures, aussi longtemps que possible, est notamment motivée par leur souhait de conserver leur liberté et leur indépendance. Par ailleurs, comme préalablement mentionné, la diminution de l'utilisation de la voiture à Fribourg n'a été observée qu'après deux décennies. Mais, malgré toutes les politiques d'aménagement et de transport mises en place, cette dernière occupe encore la première position devant les autres offres de transport ${ }^{15}$.

Les résultats de notre enquête montrent, par contre, que la voiture ne se place qu'à la troisième position du choix modal des personnes âgées de Rieselfeld. Le graphique de la figure 5 représente la distribution des moyens de transport utilisés par cette population pour répondre à leurs divers besoins de mobilité, indépendamment de la distance. On remarque assez nettement que la préférence est accordée aux transports en commun (90\%). Ensuite viennent, respectivement, la marche à pied (77\%) et la voiture (66\%) dans l'ordre des moyens de transports disponibles dans le quartier. Tout comme c'est le cas de façon générale dans la ville de Fribourg, le vélo est également un moyen de 
déplacement fortement utilisé par les seniors de Rieselfeld. Près de $60 \%$ des enquêtés y recourent, à des fréquences variables. Les autres moyens de déplacements (tels que le taxi ou la moto) mentionnés par les personnes âgées du quartier ne sont que très peu utilisés (8\%). Enfin, il est à noter que le co-voiturage et l'auto-partage ne sont quasiment pas pratiqués par les seniors interrogés.

Figure 5 : Moyens de transport les plus utilisés (en \%) par les personnes âgées à Rieselfeld

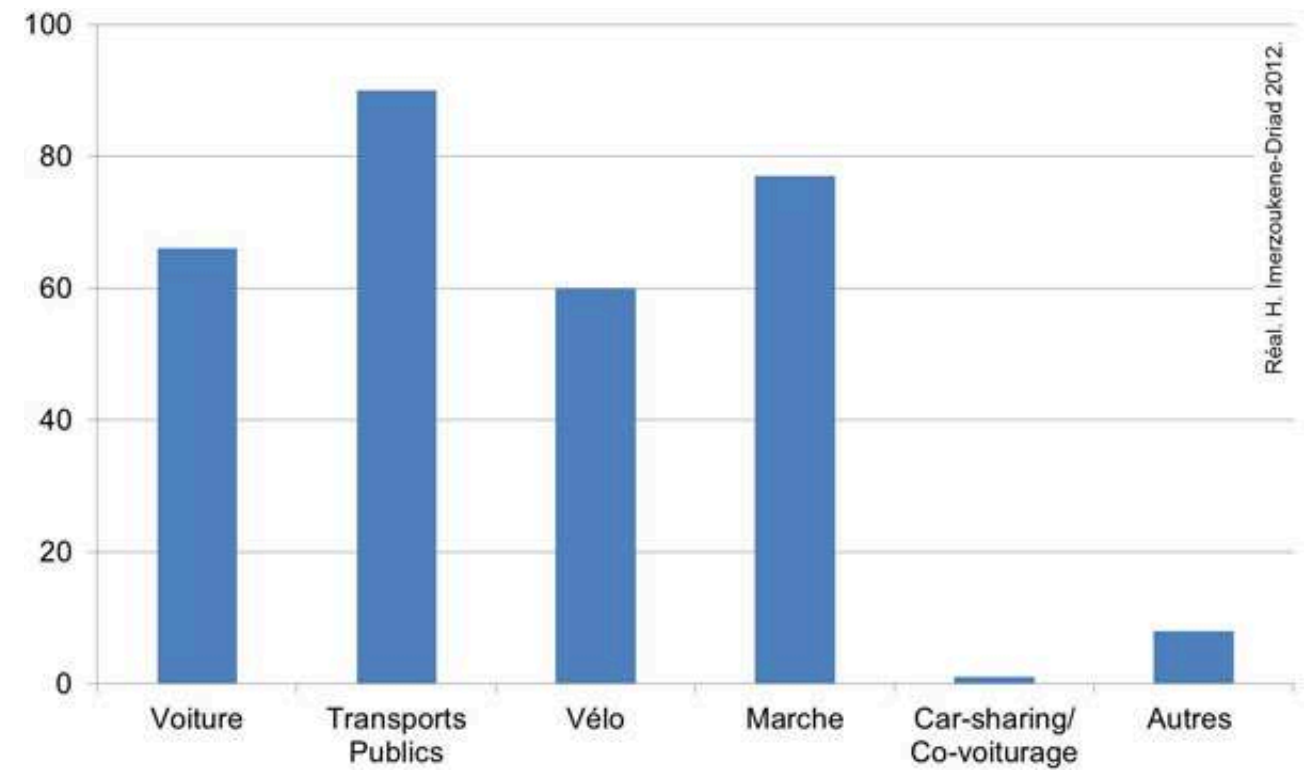

Le graphique résume les réponses à la question : « quels types de transports utilisez-vous ? », où plusieurs choix étaient possibles.

Par ailleurs, l'enquête met en évidence des utilisations préférentielles parmi les différents modes de transports en commun. En effet, les résultats obtenus à partir du croisement des différents modes de transport en commun avec la fréquence d'utilisation démontrent avec une majorité absolue que le tramway est le moyen privilégié et le plus utilisé par les personnes âgées: plus de $70 \%$ des interrogés déclarent utiliser « très souvent » le tramway.

Après un croisement des résultats entre lieux de pratique de la mobilité et fréquence de cette dernière, il s'avère, comme attendu, que la grande majorité ( $75 \%$ des enquêtés) se déplace le plus souvent dans le quartier au moins une fois par jour pour effectuer des achats ou simplement marcher. Le principal motif de déplacement, cité par près de $90 \%$ des personnes âgées à Rieselfeld, est lié aux achats. La fréquence de cette mobilité est en général quotidienne, pour les petits achats : journal, boulanger, etc. L'ouverture dans le quartier d'une supérette Lidl, et plus récemment d'une grande surface Edeka a encore concentré un peu plus le déroulement des achats quotidiens/hebdomadaires, comme le confirme Madame A. D. (61 ans) dans ce commentaire :

"Les courses, je les fais ici au marché, le samedi, c'est très sympa. En plus, c'est des produits de fermiers, donc de la région.... La voiture, je la prends quand j'achète les boissons, une fois par quinzaine peut être, pour les autres choses on a Edeka [grande surface], c'est une bonne chose ».

Par ailleurs, la promotion de longue date et à long terme de l'exercice physique, à Rieselfeld, dans le cadre de la mobilité quotidienne, a été facilitée par l'aménagement 
d'infrastructures : voiries et carrefours sécurisés, zones piétonnes, vitesse limitée à 30 $\mathrm{km} / \mathrm{h}$, bancs, parcs, etc.

48 Après le quartier, les déplacements vers le centre-ville de Fribourg occupent logiquement la deuxième place (50\%). Parmi les seniors interrogés, certains sont titulaires de la carte d'abonnement qui donne accès à tous les transports en commun. Ces déplacements sont effectués notamment pour des raisons administratives ou de santé (hôpital, clinique). Les déplacements vers les autres quartiers sont moins fréquents (30\%), et ceux vers les autres villes encore plus rares. Ceci confirme le fait que les déplacements des populations avançant en âge ont tendance à être recentrés vers le quartier, et les distances de déplacement et le temps consacré deviennent plus courts. Des résultats similaires ont été également observés à l'échelle nationale ${ }^{16}$. Sachant que les personnes âgées ont moins de déplacements obligés, la décroissance de la mobilité fréquentielle et spatiale avec l'âge est assez logique et même bien établie (Alsnih et Hensher, 2003).

\section{Mobilité durable à Rieselfeld : impression ou réalité ?}

49 Les résultats précédents montrent clairement que l'automobile n'assure pas un rôle essentiel dans la mobilité des personnes âgées du quartier. L'utilisation modérée de la voiture à Rieselfeld est corrélée, d'une part, à l'existence de structures proches et accessibles (même en fauteuils roulants ou en déambulateurs) en matière de vie sociale et culturelle (mixité fonctionnelle, maison de quartier, etc.). De plus, la place modérée de la voiture dans le quartier résulte aussi de la politique urbaine de la ville de Fribourg, basée sur la restriction de l'usage de la voiture, en même temps qu'une offre en transports en commun ou alternatifs qui se veut de qualité. Par ailleurs, pour nombre de personnes âgées interviewées, le tramway était un argument très important pour le choix de leur installation. Le succès du service de tram tourne autour de l'efficacité de ce dernier, qui est jugée (de l'avis des seniors interrogés) : «pratique, proche, confortable, et disponible».

50 En outre, le quartier dispose de trois arrêts proches les uns des autres, ce qui aide les personnes qui ont du mal à marcher à se déplacer dans le quartier. Le plan d'organisation du quartier, qui préconisait justement une distance maximale de $400 \mathrm{~m}$ entre un arrêt de tram et une habitation, facilite la tâche aux personnes âgées. La fréquence des navettes est aussi jugée raisonnable avec une durée d'attente d'environ 7 minutes en semaine. Tous ces éléments font que les transports en commun occupent une place première auprès de la population âgée de Rieselfeld. Les réseaux de transport en commun mis en place (tram, bus) forment ainsi, pour la majorité des individus, une véritable alternative en matière de mobilité. Toutefois, il n'en demeure pas moins que le recours aux transports collectifs ne va pas sans poser problème à certains usagers, notamment très âgés ou diminués physiquement. Lors de différentes réunions de quartier, des problèmes d'accessibilité dans certains tramways ont été souvent rapportés par les utilisateurs de déambulateurs ou de chaises roulantes.

51 Afin de savoir si les pratiques de transport après le départ à la retraite ont changé ou, au contraire, relèvent d'une continuité des pratiques anciennes, nous avons interrogé les personnes âgées sur les moyens de transport utilisés avant leur départ à la retraite. Il en résulte que, d'une part, la voiture était largement utilisée, quel que soit l'objectif 
du déplacement, chez plus de 50\% de cette population. D'autre part, la marche et le vélo étaient davantage associés aux activités de loisirs, pour plus de $30 \%$ des enquêtés.

Par contre, l'utilisation des transports en commun était plutôt faible $(<20 \%)$ durant la vie active. Or, nous avons précédemment vu que l'utilisation de la voiture par les seniors (majoritairement en retraite) à Rieselfeld n'occupe que la troisième place dans la classification des moyens de transport. Le croisement de ces deux résultats (avant/ après retraite) montre un changement de mobilité, avec une diminution importante de l'usage de la voiture après le passage à la retraite. $55 \%$ des seniors reconnaissent avoir opéré des changements volontaires (pour plus d'activité physique, des raisons écologiques, etc.) ou involontaires (maladies, coûts) dans leurs habitudes de déplacement. De nombreux seniors font état de l'utilisation moins fréquente de leur automobile (parfois même la vente de cette dernière) et l'utilisation subséquente de la marche et des transports en commun. Certains précisent également que la plupart de leurs activités de loisirs et d'achats sont désormais effectués dans le quartier. Le changement de comportement de mobilité a été également noté lors des interviews. C'est le cas par exemple de Monsieur A. S. (72 ans), qui précise que même si la voiture reste présente, elle est associée à des motifs spécifiques et pour des distances relativement grandes :

«Depuis que je suis ici à Rieselfeld, je marche beaucoup, la voiture c'est quand je sors avec ma femme pour aller loin, quelque part... ».

L'ensemble de ces résultats tend à supposer que les conditions morphologiques et environnementales du lieu de vie peuvent effectivement contribuer au changement. Cependant, lors de certains entretiens, quelques personnes âgées (irréductibles?) signalent que seule l'apparition d'une incapacité physique et/ou psychologique les engagerait à une adaptation de leur pratique de mobilité. Pour ces dernières, la pratique automobile relève d'une intériorisation: celle d'une autonomie et d'une indépendance acquise tout au long de la vie. Le passage à la retraite ne conduirait donc pas nécessairement à un bouleversement des pratiques de transport, mais il contribue certainement à des évolutions de ces dernières.

\section{Conclusion}

Nous avons tenté d'évaluer la mobilité des personnes âgées au sein du quartier durable de Rieselfeld. Contrairement aux données de la littérature concernant la persistance ou l'augmentation d'utilisation de l'automobile chez les personnes âgées (Rosenbloom, 2001 ; Alsnih, 2003), les résultats de notre enquête montrent que la voiture n'est pas le mode de transport privilégié qu'empruntent les seniors de ce "quartier durable ». Elle ne ressort que comme troisième choix modal, après les transports en commun (notamment le tramway) et la marche à pied. La majorité des personnes âgées se déplacent localement pour les achats, les loisirs ou à des fins médicales; du reste, les principales raisons d'«immobilité" de certains seniors sont davantage liées aux conditions de santé qu'au manque d'infrastructures.

Le système actuel de transport public à Rieselfeld reste, néanmoins, perfectible pour le rendre entièrement accessible aux personnes à mobilité réduite. De plus, les tarifs des cartes d'abonnement de transports ne semblent pas être à la portée de tous les seniors, 
ou tout au moins sont jugés non rentables pour cette catégorie d'âge, qui se déplace moins fréquemment et moins loin, comparée aux plus jeunes.

À l'issue de cette étude, il apparaît important de confirmer l'efficacité des politiques d'aménagement et de transport mises en œuvre dans le quartier de Rieselfeld et de vérifier les pratiques de mobilité de toute la population du quartier. Dans cette optique, nous avons récemment initié deux enquêtes dans les deux quartiers durables de Fribourg (Rieselfeld et Vauban) afin de pouvoir conduire des analyses comparatives et à une plus grande échelle. Ces études permettront d'avoir une meilleure lecture des comportements de mobilité des habitants des écoquartiers.

\section{BIBLIOGRAPHY}

Alsnih R., Hensher D.A., 2003, « The mobility and accessibility expectations of seniors in an aging population », Transportation Research Part A, 37, p. 903-916.

Beim M., Haag M., 2010, Freiburg's way to sustainability: the role of integrated urban and transport planning, Proceedings of Real Corp.

Buehler R., Pucher J., 2011, « Sustainable transport in Freiburg: lessons from Germany's environmental capital », International Journal of Sustainable Transportation, 5(1), p. 43-70.

Caradec V., 2007, « L'epreuve du grand âge », Retraite et société 3 (n52), p. 11-37. Consulté le 09 avril 2012, URL : www.cairn.info/revue-retraite-et-societe-2007-3-page-11.htm.

Charlot-Valdieu C., Emelianoff C., 2000, Les apports de la démarche Agenda 21 local à travers deux thèmes d'analyse : les documents d'urbanisme et l'approche quartier, Rapport pour l'ADEME et le CSTB. Coudin G., 2005, « La vieillesse n'est pas une maladie », Sciences humaines. Hors série (48), p.20-22. FitzRoy F., Smith I., 1998, « Public transport demand in Freiburg: Why did patronage double in a decade ${ }^{\circ} "$, Transport Policy 5, p. 163-173.

Hamman P., 2011, Le tramway dans la ville - Le projet urbain négocié à l'aune des déplacements, Rennes, PU Rennes, $287 \mathrm{p}$.

Hecker A., 2007, « Urbanisme, société et mobilité durable en Allemagne », Revue Géographique de l'Est, vol. 47-1.

Humpert K., 1997, Stadterweiterung: Freiburg Rieselfeld: Modell für eine wachsende Stadt, Av edition. Imerzoukene H., 2012, Déplacement des personnes âgées à l'heure de la mobilité durable : cas du quartier durable de Rieselfeld à Fribourg-en-Brisgau, Master 2 Urbanisme et Aménagement, Université de Strasbourg.

Le Breton E., 2005, Bouger pour s'en sortir. Mobilité quotidienne et intégration sociale, Paris, Armand Colin, $256 \mathrm{p}$.

Medearis D., Daseking W., 2012, « Freiburg, Germany: Germany's Eco-Capital », In Green Cities of Europe, Island Press/Center for Resource Economics, p. 65-82. 
Negron-Poblete P., 2012, « Arrimer les compétences individuelles des personnes âgées et l'accessibilité des territoires de banlieue pour une mobilité durable ", Vertigo, la revue électronique en sciences de l'environnement, mis en ligne le 02 mai 2012, consultée le 11 Septembre 2012, URL : http://vertigo.revues.org/11761

Orfeuil J.-P.., 2004, Transports, pauvretés, exclusions. Pouvoir bouger pour s'en sortir, Editions de l'Aube.

Pochet P., 1996, «La mobilité quotidienne des personnes âgées ", Gérontologie et société, 76, p. 91-106.

Rosenbloom S., 2001, « Sustainability and automobility among the elderly: An international assessment », Transportation, 28(4), p. 375-408.

Schings C., 2011, « Sozialraumanalyse Freiburg- Rieselfeld », Befragung zur Zufriedenheit der Bewohner/innen Februar, Freiburger Institut für angewandte Sozialwissenschaft .e.V (FIFAS).

Sterns R., Antenucci V., Nelson C., Glasgow N., 2003, « Public transportation: Options to maintain mobility for life », Generations, 27(2), p. 14-19.

\section{NOTES}

1. Lors de la conférence internationale de l'OCDE «Vers des transports durables », en 1996, il a été convenu que « tout être humain a droit à un accès raisonnable aux personnes, aux lieux, aux biens et aux services ainsi qu'à une information sérieuse, qui lui permette de s'orienter vers les transports durables ».

2. FRITZ: Tables d'information statistiques de Fribourg (Freiburg Informationen Tabellen Zahlen). Amt für Bürgerservice und Informationsverarbeitung, Freiburg. Abtlg. Informationsmanagement.

3. K.I.O.S.K : Contact Information Organisation Auto-aide (Kontakt Information Organisation Selbst-hilfe).

4. La question de l'étalement urbain reste toutefois controversée et les experts débattent encore des avantages respectifs des modèles de villes compactes et des villes décentralisées, sans arriver à un consensus.

5. Freiburg im Breisgau Verkehrspolitik - Transport policy (C) Stadtplanungsamt Freiburg, Abteilung Stadtarchitektur, 2006.

6. Ibid.

7. Büro R+T (2002): Verkehrsentwicklungsplan Freiburg. Teil A: Problemanalyse. http:// www.freiburg.de/download/verkehrsentwicklungsplan/vep_analysebericht.pdf

8. Umweltpolitik in Freiburg, Stadt Freiburg, 2012, p. 18.

9. Freiburger Institut für angewandte Sozialwissenschaft .e.V.

10. FR-ITZ, Amt für Bürgerservice und Informationsverarbeitung, Freiburg. Abtlg. Informationsmanagement.

11. Idem.

12. Statistiques «en ligne» de la ville de Fribourg (Anteil der Wohnbevölkerung ab 60 Jahren Rieselfeld).

13. ÄwiR : Älter werden im Rieselfeld - Vieillir à Rieselfeld.

14. Rapport de la $112^{\mathrm{e}}$ Table ronde d'économie des transports et vieillissement de la population Conférence européenne des ministres des transports CEMT, Paris, 1998.

15. Freiburg im Breisgau Verkehrspolitik - Transport policy (c) Stadtplanungsamt Freiburg, Abteilung Stadtarchitektur, 2006. 
16. W. Brög, 1998, dossier Allemagne dans le rapport de la $112^{\mathrm{e}}$ Table ronde d'économie des transports et vieillissement de la population Conférence européenne des ministres des transports CEMT, Paris, p.45-147.

\section{ABSTRACTS}

This paper discusses the adaptability of the "sustainable neighborhood" Rieselfeld in Freiburg im Breisgau (Germany) to seniors. From a field survey on their mobility behavior, we analyzed the spatial accessibility in view to identify potential barriers encountered during the use of various means of transport. The main findings reflect the localized nature of most trips. Moreover, even if the car is still quite present, seniors prefer soft and public modes of transportation, especially the tram. These results may be reported, on the one hand, to the integrated policies of land use and transport developed in the citywide; and on the other hand, to the social, cultural and natural environment of the district. These factors appear to allow seniors to remain longer independent, with all the benefits that may arise.

Cet article questionne l'adaptabilité du « quartier durable » de Rieselfeld à Fribourg-en-Brisgau (Allemagne) aux seniors. À partir d'une enquête de terrain menée sur les comportements de mobilité de ces derniers, nous avons analysé l'accessibilité spatiale en cherchant à identifier les barrières potentielles rencontrées lors de l'utilisation de différents moyens de déplacement. Les principales conclusions reflètent la nature localisée de la plupart des déplacements. Par ailleurs, même si la voiture reste assez présente, les seniors privilégient davantage les modes doux et les transports en commun, en particulier le tramway. Ces résultats peuvent être rapportés aux politiques intégrées d'aménagement du territoire et des transports développées à l'échelle de la ville, d'une part ; et, d'autre part, à l'environnement social, culturel et naturel du quartier. Ces facteurs semblent permettre aux personnes âgées de rester plus longtemps autonomes, avec tous les avantages qui peuvent en découler.

Dieser Beitrag diskutiert, inwiefern das "nachhaltige Quartier" Rieselfeld in Freiburg im Breisgau (Deutschland) der Situation von älteren Menschen gerecht wird. Auf der Grundlage einer Befragung zum Mobilitätsverhalten der Älteren, die vor Ort durchgeführt wurde, haben wir die räumliche Erreichbarkeit untersucht und dabei mögliche Barrieren identifiziert, die bei der Nutzung verschiedener Verkehrsmittel auftreten. Aus den wichtigsten Ergebnissen geht hervor, mit Hilfe welcher Verkehrsmittel die meisten Wege zurückgelegt werden. Es zeigt sich, dass ungeachtet einer weiterhin recht starken Präsenz des Autos von den Senioren vor allem sanfte Mobilität sowie öffentliche Verkehrsmittel und insbesondere die Straßenbahn bevorzugt werden. Diese Ergebnisse beziehen sich einerseits auf die Raumordnung und Verkehrsentwicklung auf der Ebene der gesamten Stadt und andererseits auf die soziale, kulturelle und natürliche Umwelt im Quartier. Diese Faktoren ermöglichen es älteren Menschen, länger unabhängig zu bleiben und alle damit verbundenen Vorteile zu nutzen. 
INDEX

Schlüsselwörter: ältere Menschen, Alterung, Freiburg-Rieselfeld, Mobilität, Nachhaltiges Quartier, Stadtpolitik

Keywords: aging, elderly, Freiburg-Rieselfeld, mobility, sustainable neighborhood, urban policy Mots-clés: Fribourg-Rieselfeld, mobilité, politique de la ville, quartier durable, seniors, vieillissement

\section{AUTHORS}

\section{HASSINA IMERZOUKENE DRIAD}

Faculté des Sciences sociales, UMR SAGE, CNRS-Université de Strasbourg - Fakultät für Umwelt und Natürliche Ressourcen, Albert-Ludwigs-Universität - Freiburg hassina.imerzoukene@geographie.uni-freiburg.de

\section{PHILIPPE HAMMAN}

Faculté des Sciences sociales, UMR SAGE, CNRS-Université de Strasbourg

\section{TIM FREYTAG}

Fakultät für Umwelt und Natürliche Ressourcen, Albert-Ludwigs-Universität Freiburg 